\title{
Variation of Chemical Compounds of Naturally and Artificially Formed Agarwood Resins in Gyrinops walla
}

\author{
de Alwis W.N.H.1*, Subasinghe S.M.C.U.P. ${ }^{1}$, Hettiarachchi D.S. ${ }^{2}$ \\ ${ }^{1}$ Department of Forestry and Environmental Science, University of Sri Jayewardenepura, Sri Lanka \\ ${ }^{2}$ Wescorp Group of Companies, Western Australia \\ *hasinide26@gmail.com
}

\begin{abstract}
Gyrinops walla, a member of Thymelaeaceae family produces valuable agarwood resin used for religious, cultural and medicinal purposes in many countries. It is formed as a self-defense mechanism to any stress caused physical, chemical or biological agents. Since a comparison of agarwood formed due to natural and artificial causes was not done previously, the present study was conducted to fulfill that objective. For this reason, $150 \mathrm{G}$. walla trees growing in nine locations (Horawala, Kalatuwawa, Karandana, Maliboda, Neboda, Yagirala, Mirigama, Kalawana and Suriyakanda) of wet zone, Sri Lank were selected for collecting agarwood resins formed due to natural causes. Medium size G. walla trees growing in Horawala and Kalawana were selected for formation of agarwood resins in artificial manner. Insertions of iron nails, galvanised tubes, polyvinyl chloride tubes and polypropylene tubes were used as the mechanical method; sodium chloride and sodium bi-carbonate were used as the chemical method and forest mushroom mycelia was used as the biological method to form agarwood resins. Three replicates were used for each agent and agarwood tissues were collected after 12 months of inoculation. Solvent extraction was used to extract agarwood resins and constituent analysis was done by GC-FID method. It was possible to identify 21 compounds belonging to seven classes, viz., agarospirol, vetispirane, selinene, cardinane, guaiene, eremophilane, 2-(2phenylehtyl)-chromone and fatty acids from naturally formed agarwood resins. However, 28 compounds were present in the resins formed due to artificial methods belonging to nine classes. Among them agarofuran, and valencane were only found in the artificially formed resins. Agarospirol and agarofuran are the most important compounds that produce the characteristic aroma of agarwood resins. However, agarofuran was not observed from the naturally formed agarwood resins. All compounds identified in this study are present in the agarwood resins of Aquilaria species grown in Southeast Asian countries. The results confirmed that artificially formed agarwood in $G$. walla chemically bears the same quality of naturally produced agarwood.
\end{abstract}

Keywords: Agarwood, Artificial inoculation, Chemical compounds, Gyrinops walla, Sri Lanka

Acknowledgement: Authors acknowledge joint research grant provided by National Research Council and Sadaharitha Plantations Limited (NRC-PPP- 12-57) and the assistance of Centre for Forestry and Environment, University of Sri Jayewardenepura.

Proceedings of the $22^{\text {nd }}$ International Forestry and Environment Symposium 2017 of the Department of Forestry and Environmental Science, University of Sri Jayewardenepura, Sri Lanka 